\title{
ON CONSTRUCTING BANACH SPACES WITH NO UNCONDITIONAL BASIS
}

\author{
RYSZARD KOMOROWSKI
}

(Communicated by William J. Davis)

AbStract. There exists a weak Hilbert space with no unconditional basis.

The aim of this note is to show a rather general construction of Banach spaces with no unconditional basis. As a corollary, one obtains a weak Hilbert space with no unconditional basis, thus answering a question raised some years ago by several authors (cf., e.g., [CS, P]). Our approach is based on techniques first introduced by Johnson, Lindenstrauss, and Schechtman in [JLS] for the study of the Kalton-Peck space [KP]. These techniques were refined by Ketonen [K] and generalized further by Borzyszkowski [B]. The novelty of the present general approach consists of the use of a simple interpolation trick, which is nevertheless strong enough to allow for the construction of a weak Hilbert space which contains a subspace with no unconditional basis.

\section{THE MAIN CONSTRUCTION}

The standard notation from the Banach space theory used throughout this paper can be found, for example, in $[\mathrm{P}, \mathrm{TJ}]$.

Let us recall that, if $\left(Z_{k}\right)_{k=1}^{\infty}$ is a family of finite-dimensional subspaces of a Banach space $X$, then the unconditional constant of $\left(Z_{k}\right)_{k=1}^{\infty}$, denoted by unc $\left(Z_{k}\right)_{k=1}^{\infty}$, is the infimum of numbers $c>0$ such that, for all finite sequences of vectors $\left(x_{k}\right)_{k}$, with $x_{k} \in Z_{k}$, and for all choices of signs $\left(\varepsilon_{k}\right)_{k}$, the following holds:

$$
\left\|\sum_{k} \varepsilon_{k} x_{k}\right\| \leq c\left\|\sum_{k} x_{k}\right\| .
$$

If $\operatorname{unc}\left(Z_{k}\right)_{k=1}^{\infty}$ is finite, we call $\left(Z_{k}\right)_{k=1}^{\infty}$ an unconditional decomposition.

A basis $\left(e_{i}\right)_{i=1}^{\infty}$ in a Banach space $X$ is 1 -conditional if unc $\left(\operatorname{span}\left\{e_{i}\right\}_{i=1}^{\infty}\right)=1$. If $A$ is a set of positive integers, we denote $\operatorname{span}\left(\left\{e_{i}\right\}_{i \in A}\right)$ in $X$ by $\left.X\right|_{A}$.

Throughout this paper we fix an interpolation functor which to any $0<\theta<1$ and any interpolation couple of Banach spaces $\left(X_{0}, X_{1}\right)$, with norms $\|\cdot\|_{0}$ and $\|\cdot\|_{1}$ respectively, corresponds the space $\left(X_{0}, X_{1}\right)_{\theta}$, with the norm $\|\cdot\|_{\theta}$ such that:

Received by the editors January 5, 1992.

1991 Mathematics Subject Classification. Primary 46B99. 
(a) whenever $\left(X_{0}, X_{1}\right)$ and $\left(Y_{0}, Y_{1}\right)$ are two interpolation couples and $T$ is a linear operator such that $\left\|T: X_{j} \rightarrow Y_{j}\right\|=M_{j}$, for $j=0,1$, then one has

$$
\left(X_{0}, X_{0}\right)_{\theta}=X_{0} \text { and }\left\|T:\left(X_{0}, X_{1}\right)_{\theta} \rightarrow\left(Y_{0}, Y_{1}\right)_{\theta}\right\| \leq M_{0}^{1-\theta} M_{1}^{\theta} ;
$$

(b) whenever $\left(X_{0}, X_{1}\right)$ is an interpolation couple and $\left(e_{i}\right)_{i=1}^{\infty}$ is a 1-unconditional basis in both $X_{0}$ and $X_{1}$ and $A$ is a set of positive integers, then

$$
\left(\left.X_{0}\right|_{A},\left.X_{1}\right|_{A}\right)_{\theta}=\left.\left(X_{0}, X_{1}\right)_{\theta}\right|_{A} .
$$

It is well known (cf., e.g., [BL]) that the complex method satisfies conditions (a) and (b). The real $K$ - and $J$-methods satisfy (b), but (a) holds only up to a factor depending on the parameters of the method. However, by multiplying the interpolation norms by suitable constants, one can actually satisfy condition (a) as well.

Condition (a) implies the following: for all $x \in X_{0} \cap X_{1}$ one has

$$
\|x\|_{\theta} \leq\|x\|_{0}^{1-\theta}\|x\|_{1}^{\theta} .
$$

Let us observe the following fact crucial for further arguments.

Remark 1. Let $X_{0}=X_{1}=\mathbf{R}^{n}$ be two Banach spaces with norms $\|\cdot\|_{0}$ and $\|\cdot\|_{1}$, respectively. Let $\|$ id: $X_{0} \rightarrow X_{1} \|=D$, and let $z_{0} \in \mathbf{R}^{n}$ be such that $\left\|z_{0}\right\|_{0}=1$ and $\left\|z_{0}\right\|_{1}=D$. Then $\left\|z_{0}\right\|_{\theta}=D^{\theta}$.

Indeed, since $\|$ id: $\left(X_{0}, X_{1}\right)_{\theta} \rightarrow X_{1} \| \leq D^{1-\theta}$, we get, by (1),

$$
D=\left\|z_{0}\right\|_{1} \leq D^{1-\theta}\left\|z_{0}\right\|_{\theta} \leq D^{1-\theta}\left\|z_{0}\right\|_{0}^{1-\theta}\left\|z_{0}\right\|_{1}^{\theta}=D .
$$

The main theorem of this section is

Theorem 1. Let $\left(X_{0},\|\cdot\|_{0}\right)$ and $\left(X_{1},\|\cdot\|_{1}\right)$ be two Banach spaces of finite cotype, let $X_{1} \subset X_{0}$, and let $\|x\|_{0} \leq\|x\|_{1}$ for $x \in X_{1}$. Let $\left(e_{i}\right)_{i=1}^{\infty}$ be a normalized 1-unconditional basis for $\left(X_{0},\|\cdot\|_{0}\right)$ and $\left(X_{1},\|\cdot\|_{1}\right)$. Assume that, for every infinite set $A$ of positive integers, there exists a sequence $\left(a_{k}\right)_{k \in A}$ of scalars such that the series $\sum_{k \in A} a_{k} e_{k}$ is convergent in $X_{0}$ and divergent in $X_{1}$. Then for every increasing sequence $\left(\theta_{i}\right)_{i=1}^{\infty}$ in $(0,1)$, and for every $1 \leq p \leq \infty$, the space $\left(\sum \bigoplus\left(X_{0}, X_{1}\right)_{\theta_{i}}\right)_{p}$ has a subspace $Y$ which has a basis but has no unconditional basis.

Actually, the subspace $Y$ even does not have the local unconditional structure.

The proof is based on a modification of the following criterion, due to Ketonen $[\mathrm{K}]$ and Borzyszkowski $[\mathrm{B}]$.

Proposition 1. Let $Y$ be a Banach space of finite cotype with a 2-dimensional unconditional decomposition $Y=\overline{\operatorname{span}\left(\left\{Z_{k}\right\}_{k=1}^{\infty}\right)}$. Let $Z_{k}=\operatorname{span}\left\{x_{k}, y_{k}\right\}$ for $k=1,2, \ldots$, and assume there exists $C \geq 1$ such that, for every $\alpha, \beta \in \mathbf{R}$ and $k=1,2, \ldots$,

(i) $\max (|\alpha|,|\beta|) \leq\left\|\alpha x_{k}+\beta y_{k}\right\| \leq C(|\alpha|+|\beta|)$.

If $\operatorname{unc}(Y)<\infty$, then there exists an infinite set $A$ of positive integers, $a$ bounded linear operator $T: \overline{\operatorname{span}\left(\left\{Z_{k}\right\}_{k \in A}\right)} \rightarrow \overline{\operatorname{span}\left(\left\{Z_{k}\right\}_{k \in A}\right)}$, and $a, b, c, d \in$ $\mathbf{R}$ such that:

(ii) $T\left(\sum_{k \in A} \alpha_{k} x_{k}+\beta_{k} y_{k}\right)=\sum_{k \in A}\left(a \alpha_{k}+b \beta_{k}\right) x_{k}+\left(c \alpha_{k}+d \beta_{k}\right) y_{k}$;

(iii) for every $k \in A$ and for every scalar $\lambda \in \mathbf{R}$, one has $\left\|(T-\lambda \mathrm{id}) \mid z_{k}\right\| \geq C_{1}$, where $C_{1}>0$ is an absolute constant. 
In fact, there is a function $f$ such that if $Y$ is of cotype $q$ with the cotype $q$ constant $K_{1}, K_{2}=\operatorname{unc}\left\{Z_{k}\right\}_{k=1}^{\infty}$, and $C$ is a constant from (i), then $\|T\| \leq$ $f\left(C, q, K_{1}, K_{2}, \operatorname{unc}(Y)\right)$.

We will use Proposition 1 by constructing, in the situation of Theorem 1 , a subspace $Y$ which does not admit a bounded operator $T$ as above. This is done in the main technical proposition which states:

Proposition 2. Let $X_{0}, X_{1},\left(\theta_{i}\right)$, and $p$ be as in Theorem 1. Then there exists a basic sequence $x_{1}, y_{1}, x_{2}, y_{2}, \ldots$ in $\left(\sum \bigoplus\left(X_{0}, X_{1}\right)_{\theta_{i}}\right)_{p}$ and $C \geq 1$ such that, for every $\alpha, \beta \in \mathbf{R}$ and $k=1,2, \ldots$, one has

(i) $\max (|\alpha|,|\beta|) \leq\left\|\alpha x_{k}+\beta y_{k}\right\| \leq C(|\alpha|+|\beta|)$;

(ii) the decomposition $\left(Z_{k}\right)_{k=1}^{\infty}$, where $Z_{k}=\operatorname{span}\left\{x_{k}, y_{k}\right\}$ for $k=1,2, \ldots$, is unconditional.

Moreover, for every infinite set $A$ of positive integers one has

(iii) the linear operator $\sum_{k \in A} \alpha_{k} x_{k} \rightarrow \sum_{k \in A} \alpha_{k} y_{k}$, acting from $\operatorname{span}\left(\left\{x_{k}\right\}_{k \in A}\right)$ to $\operatorname{span}\left(\left\{y_{k}\right\}_{k \in A}\right)$, is not bounded;

(iv) the linear operator $\sum_{k \in A} \alpha_{k} y_{k} \rightarrow \sum_{k \in A} \alpha_{k} x_{k}$, acting from $\operatorname{span}\left(\left\{y_{k}\right\}_{k \in A}\right)$ to $\operatorname{span}\left(\left\{x_{k}\right\}_{k \in A}\right)$, is not bounded;

(v) the projection $\sum_{k \in A} \alpha_{k} x_{k}+\beta_{k} y_{k} \rightarrow \sum_{k \in A} \alpha_{k} x_{k}$, acting from $\operatorname{span}\left(\left\{Z_{k}\right\}_{k \in A}\right)$ to $\operatorname{span}\left(\left\{x_{k}\right\}_{k \in A}\right)$, is not bounded.

Assuming the truth of Propositions 1 and 2 we can prove Theorem 1.

Proof of Theorem 1. Let $\left(x_{k}\right)_{k=1}^{\infty}$ and $\left(y_{k}\right)_{k=1}^{\infty}$ be as in Proposition 2. Put $Z_{k}=\operatorname{span}\left\{x_{k}, y_{k}\right\}$ and $Y=\operatorname{span}\left(\left\{Z_{k}\right\}_{k=1}^{\infty}\right)$. Suppose that $\operatorname{unc}(Y)<\infty$. By Proposition 1 there is an infinite set $A$ of positive integers and a bounded operator $T: \overline{\operatorname{span}\left(\left\{Z_{k}\right\}_{k=1}^{\infty}\right)} \rightarrow \overline{\operatorname{span}\left(\left\{Z_{k}\right\}_{k=1}^{\infty}\right)}$ such that (i), (ii), and (iii) hold.

Assume $b \neq 0$. Suppose that $\sum_{k \in A} \beta_{k} y_{k}$ converges. Since $T$ is continuous, $\sum_{k \in A} b \beta_{k} x_{k}+d \beta_{k} y_{k}$ converges too. It follows that $\sum_{k \in A} \beta_{k} x_{k}$ converges, which contradicts Proposition 2(iv). Similarly we get $c=0$. Hence by Proposition 1 (iii) $a \neq d$. It follows that the projection $\sum_{k \in A} \alpha_{k} x_{k}+\beta_{k} y_{k} \rightarrow \sum_{k \in A} \alpha_{k} x_{k}$ is continuous, but this contradicts Proposition $2(\mathrm{v})$.

Therefore, $\operatorname{unc}(Y)=\infty$.

We pass now to the proofs of Propositions 1 and 2 .

Proof of Proposition 1. By [B, Proposition A], there is a constant $D<\infty$ and operators $T_{n}: Y \rightarrow Y$, for $n=1,2, \ldots$, such that:

$$
\begin{gathered}
T_{n}\left(Z_{k}\right) \subset Z_{k} \text { for each } k, \\
\left\|T_{n}\right\| \leq D, \\
\left\|\left.\left(T_{n}-\lambda \mathrm{id}\right)\right|_{Z_{k}}\right\| \geq \frac{1}{8} \quad \text { for every } \lambda \in \mathbf{R}, k=1,2, \ldots, n .
\end{gathered}
$$

Let $A_{n, k}$, for $n=1,2, \ldots, k=1,2, \ldots, n$, be the family of $2 \times 2$ matrices of operators $T_{n} \mid z_{k}$ with respect to the basis $\left\{x_{k}, y_{k}\right\}$. By (3) and condition (i), the coefficients of $A_{n, k}$ are bounded. Hence, by the Cantor diagonal process, we can extract an increasing sequence $\left(n_{j}\right)_{j=1}^{\infty}$ of integers such that for every $k$ the $\operatorname{limit}_{\lim _{j \rightarrow \infty}} A_{n_{j}, k}=A_{k}$ exists. Let

$$
A_{k}=\left[\begin{array}{ll}
a_{k} & b_{k} \\
c_{k} & d_{k}
\end{array}\right] \text {. }
$$


Set

$$
T_{1}\left(\sum_{k=1}^{\infty} \alpha_{k} x_{k}+\beta_{k} y_{k}\right)=\sum_{k=1}^{\infty}\left(a_{k} \alpha_{k}+b_{k} \beta_{k}\right) x_{k}+\left(c_{k} \alpha_{k}+d_{k} \beta_{k}\right) y_{k}
$$

By the Banach-Steinhaus theorem, $\left\|T_{1}\right\| \leq D$. Moreover,

$$
\|\left.\left(T_{1}-\lambda \text { id }\right)\right|_{Z_{k}} \| \geq \frac{1}{8} \text { for every } \lambda \in \mathbf{R}, k=1,2, \ldots .
$$

Fix $0<C_{0}<1$ to be defined later, and choose a subsequence $\left(k_{j}\right)_{j=1}^{\infty}$ such that

$$
\max \left(\left|a_{k_{j}}-a\right|,\left|b_{k_{j}}-b\right|,\left|c_{k_{j}}-c\right|,\left|d_{k_{j}}-d\right|\right)<C_{0} 2^{-j}
$$

for $j=1,2, \ldots$ Of course, such $a, b, c, d$ exist by compactness. Put $A=\left\{k_{1}, k_{2}, \ldots\right\}$, and define $T: \operatorname{span}\left(\left\{Z_{k}\right\}_{k \in A}\right) \rightarrow \operatorname{span}\left(\left\{Z_{k}\right\}_{k \in A}\right)$ by

$$
T\left(\sum_{k \in A} \alpha_{k} x_{k}+\beta_{k} y_{k}\right)=\sum_{k \in A}\left(a \alpha_{k}+b \beta_{k}\right) x_{k}+\left(c \alpha_{k}+d \beta_{k}\right) y_{k} .
$$

Using condition (i) and the assumption that $\left(Z_{k}\right)_{k=1}^{\infty}$ is an unconditional decomposition of $Y$, it follows that $\left\|\left.\left(T_{1}-T\right)\right|_{\operatorname{span}\left(\left\{Z_{k}\right\}_{k \in A}\right)}\right\|$ is bounded; so, $T$ is bounded and hence it can be extended to the bounded operator on

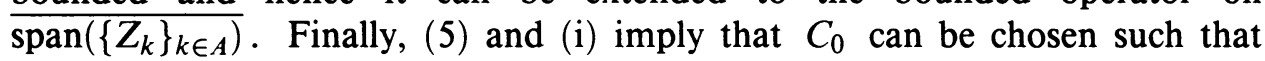
(iii) holds.

Before we start the proof of Proposition 2 we need the following lemma.

Lemma 1. Let $\left(e_{i}\right)_{i=1}^{\infty}$ be a normalized 1-unconditional basis for two Banach spaces $Y_{0}$ and $Y_{1}$. Suppose that $\left\|\mathrm{id}: Y_{0} \rightarrow Y_{1}\right\|=\infty$. Then for every $D \geq 1$ there exists a positive integer $n_{0}$ such that

$$
D \leq\left\|\mathrm{id}:\left.\left.Y_{0}\right|_{A_{0}} \rightarrow Y_{1}\right|_{A_{0}}\right\| \leq 2 D
$$

where $A_{0}=\left\{1,2, \ldots, n_{0}\right\}$.

Proof. For $n=1,2, \ldots$, put $A_{n}=\{1,2, \ldots, n\}$ and $d_{n}=\|$ id: $\left.Y_{0}\right|_{A_{n}} \rightarrow$ $\left.Y_{1}\right|_{A_{n}} \|$. Observe that $d_{n}$ is an increasing sequence and $\lim _{n \rightarrow \infty} d_{n}=\infty$. Now, the lemma follows from the inequality $d_{n+1} \leq 1+d_{n}$.

Proof of Proposition 2. First, observe that there exist two sequences $\left(D_{n}\right)_{n=3}^{\infty}$ and $\left(\varepsilon_{n}\right)_{n=3}^{\infty}$ such that $D_{n} \geq 1,0<\varepsilon_{n}<1$, and that, for every $n \geq 3$,

$$
\varepsilon_{n}\left(8 D_{n}^{\theta_{n-1}-\theta_{n}}+4 \varepsilon_{n+1} D_{n}\right)^{-1}>n \text { and } \varepsilon_{n+1}<\varepsilon_{n} / 2 \text {. }
$$

This can be shown by an easy induction, since $\theta_{n-1}-\theta_{n}<0$.

Set $\varepsilon_{1}=\varepsilon_{2}=1$. By $\left(e_{i k}\right)_{k=1}^{\infty}$ denote the normalized 1-unconditional basis in $\left(X_{0}, X_{1}\right)_{\theta_{i}}$ for $i=1,2, \ldots$. Fix any $a_{i}, b_{i} \in\{0,1\}$, for $i=1,2, \ldots$, such that $a_{1}=b_{1}=1, b_{1}=a_{2}=0$, and

$$
\begin{gathered}
a_{i}=b_{i}=1 \text { infinitely many times, } \\
a_{i}=0 \text { and } b_{i}=1 \text { infinitely many times, } \\
a_{i}=1 \text { and } b_{i}=0 \text { infinitely many times. }
\end{gathered}
$$

Then set

$$
x_{k}=\sum_{i=1}^{\infty} \varepsilon_{i} a_{i} e_{i k}, \quad y_{k}=\sum_{i=1}^{\infty} \varepsilon_{i} b_{i} e_{i k}
$$


To see (ii) it is enough to observe that, for $k \neq k^{\prime}$, the spaces $Z_{k}$ and $Z_{k^{\prime}}$ have disjoint supports. Since $\left\|x_{k}\right\|<2,\left\|y_{k}\right\|<2$, and $\left\|\alpha x_{k}+\beta y_{k}\right\| \geq$ $\left\|\alpha e_{1 k}+\beta e_{2 k}\right\| \geq \max (|\alpha|,|\beta|)$, we obtain (i). Now, it is easy to see that $x_{1}, y_{1}, x_{2}, y_{2}, \ldots$ is a basic sequence.

Next, let us prove part (iii). Let $A$ be an infinite set of positive integers. Fix an arbitrary $m \geq 3$ such that $b_{m}=1$ and $a_{m}=0$. By the assumption on $A$ and by Lemma 1 , with $D=D_{m}$, we conclude that there exists a positive integer $n_{0}$ such that for $d_{0}=\left\|\mathrm{id}:\left.\left.X_{0}\right|_{A_{0}} \rightarrow X_{1}\right|_{A_{0}}\right\|$ where $A_{0}=A \cap\left\{1,2, \ldots, n_{0}\right\}$, one has

$$
D_{m} \leq d_{0} \leq 2 D_{m} .
$$

Choose $\left(\beta_{k}\right)_{k \in A_{0}}$ such that $\left\|\sum_{k \in A_{0}} \beta_{k} e_{k}\right\|_{0}=1$ and $\left\|\sum_{k \in A_{0}} \beta_{k} e_{k}\right\|_{1}=d_{0}$. Denote $z_{0}^{i}=\sum_{k \in A_{0}} \beta_{k} e_{i k} \in\left(X_{0}, X_{1}\right)_{\theta_{i}}$. By property (c) and Remark 1, we obtain $\left\|z_{0}^{i}\right\|_{\theta_{i}}=d_{0}^{\theta_{i}}$, for $i=1,2, \ldots$ Thus, by (10), $D_{m}^{\theta_{i}} \leq\left\|z_{0}^{i}\right\|_{\theta_{i}} \leq 2 D_{m}^{\theta_{i}}$.

We have

$$
\begin{aligned}
\frac{\left\|\sum_{k \in A_{0}} \beta_{k} y_{k}\right\|}{\left\|\sum_{k \in A_{0}} \beta_{k} x_{k}\right\|} & =\frac{\left\|\sum_{i=1}^{\infty} \varepsilon_{i} b_{i} \sum_{k \in A_{0}} \beta_{k} e_{i k}\right\|}{\left\|\sum_{i \neq m} \varepsilon_{i} a_{i} \sum_{k \in A_{0}} \beta_{k} e_{i k}\right\|} \\
& \geq \frac{\varepsilon_{m}\left\|z_{0}^{m}\right\|_{\theta_{m}}}{\sum_{i \neq m} \varepsilon_{i}\left\|z_{0}^{i}\right\|_{\theta_{i}}} \geq \frac{\varepsilon_{m} D_{m}^{\theta_{m}}}{2\left(\sum_{i \neq m} \varepsilon_{i} D_{m}^{\theta_{i}}\right)} \\
& \geq \frac{\varepsilon_{m} D_{m}^{\theta_{m}}}{8 D_{m}^{\theta_{m-1}}+4 \varepsilon_{m+1} D_{m}} \geq \varepsilon_{m}\left(8 D_{m}^{\theta_{m-1}-\theta_{m}}+4 \varepsilon_{m+1} D_{m}\right)^{-1}>m .
\end{aligned}
$$

Since, by (8), $m$ can be taken arbitrarily large, the obtained estimate implies (iii).

Similarly, using (9), we obtain (iv). To see (v), fix an arbitrary $m \geq 3$ such that $b_{m}=a_{m}=1$. As in the proof of (iii), there exists a finite subset $A_{0}$ of $A$ and a sequence $\left(\beta_{k}\right)_{k \in A_{0}}$ such that for $z_{0}^{i}=\sum_{k \in A_{0}} \beta_{k} e_{i k} \in\left(X_{0}, X_{1}\right)_{\theta_{i}}$ one has

$$
\frac{\left\|\sum_{k \in A_{0}} \beta_{k} x_{k}\right\|}{\left\|\sum_{k \in A_{0}} \beta_{k}\left(x_{k}-y_{k}\right)\right\|} \geq \frac{\varepsilon_{m}\left\|z_{0}^{m}\right\|_{\theta_{m}}}{\sum_{i \neq m} \varepsilon_{i}\left\|z_{0}^{i}\right\|_{\theta_{i}}}>m \text {. }
$$

Therefore, $(\mathrm{v})$ is concluded by (7).

Letting $X_{j}=l_{q_{j}}$ for $j=0,1$ and $1 \leq q_{1}<q_{0} \leq \infty$ and using a suitable interpolation functor, we get $\left(X_{0}, X_{1}\right)_{\theta}=l_{q}$ where $1 / q=(1-\theta) / q_{0}+\theta / q_{1}$. As an immediate consequence of Theorem 1 we obtain

Corollary 1. For any decreasing sequence $\left(q_{i}\right)_{i=1}^{\infty}$ in $(1, \infty)$ and any $1 \leq p \leq$ $\infty$, the space $\left(\sum \bigoplus l_{q_{i}}\right)_{p}$ has a subspace with no unconditional basis.

Taking arbitrary $1 \leq p<q_{i}<2$ and observing that the space $\left(\sum \bigoplus l_{q_{i}}\right)_{p}$ is then isomorphic to a subspace of $L_{p}([0,1])$, we reconstruct Ketonen's result.

Corollary 2. For any $1 \leq p<2$, the space $L_{p}([0,1])$ has a subspace with no unconditional basis.

\section{WEAK Hilbert SPACES Without aN UNCONDITIONAL BASIS}

In this section we shall use some notation and facts contained in [P]. The main result of this section is 
Theorem 2. There exists a weak Hilbert space with no unconditional basis.

Let $T$ be any version of the 2-convexified Tsirelson space, presented in [CS; $\mathrm{P}$, Chapter 13]. To fix attention, we set, in the notation from [P], $T=X_{\delta}$, with $\delta=2^{-1 / 2}$. Then $T$ is a weak Hilbert space and the standard unit vector basis is normalized and 1-unconditional in $T$.

An interpolation functor required in this section will be assumed to satisfy, besides conditions (a) and (b) of $\S 1$, also the following condition:

(c) whenever $\left(X_{0}, X_{1}\right)$ is an interpolation couple, $\left(l_{2}\left(X_{0}\right), l_{2}\left(X_{1}\right)\right)_{\theta}=$ $l_{2}\left(\left(X_{0}, X_{1}\right)_{\theta}\right)$.

It is well known (cf., e.g., [BL]) that the complex method satisfies (c). An appropriate modification of $K$-and $J$-functionals shows that real interpolation methods, with suitably chosen parameters, also satisfy (c).

We require the following proposition, based on some rather general interpolation properties of $T$. To make the notation more compact, we set, for $0<\theta<1, T_{\theta}=\left(T, \ell_{2}\right)_{\theta}$.

Proposition 3. There exists an increasing sequence $\left(\theta_{k}\right)_{k=1}^{\infty}$ in $(0,1)$ such that the space $\left(\sum_{k=1}^{\infty} \bigoplus T_{\theta_{k}}\right)_{2}$ is a weak Hilbert space.

Now, the proof of Theorem 2 follows immediately from Theorem 1, Proposition 3, and the well-known fact that no subsequence of the basis of $T$ is equivalent to the standard unit vector basis in $\ell_{2}$ (see, e.g., $[C S]$ or $[\mathrm{P}]$ ).

Proof of Proposition 3. Fix a positive integer $n$, and let $J_{n}=\{n+1, n+2, \ldots\}$.

First, we shall show that there exists an increasing sequence $\theta_{k}$ in $(0,1)$ such that, if $F$ is an $\left(n 2^{k}\right)$-dimensional subspace of $\left.T_{\theta_{k}}\right|_{J_{n}}$, then $d\left(F, \ell_{2}^{n 2^{k}}\right) \leq 2$.

Indeed, it is easy to see from the definition of the norm on $T$ (cf., e.g., [P, (3.9) and (3.10)]) that arbitrary vectors $y_{1}, \ldots, y_{n 2^{k}}$ in $\left.T\right|_{J_{n}}$ satisfy

$$
2^{-(k+1) / 2}\left(\sum_{s=1}^{n 2^{k}}\left\|y_{s}\right\|^{2}\right)^{1 / 2} \leq\left\|\left(\sum_{s=1}^{n 2^{k}}\left|y_{s}\right|^{2}\right)^{1 / 2}\right\| \leq\left(\sum_{s=1}^{n 2^{k}}\left\|y_{s}\right\|^{2}\right)^{1 / 2} .
$$

This yields, as in [P, Lemma 13.3], that for all orthogonal $n 2^{k} \times n 2^{k}$ matrices $\left(a_{i s}\right)$ and for all $n 2^{k}$-tuples $\left(y_{s}\right)_{s=1}^{n 2^{k}}$ in $\left.T\right|_{J_{n}}$ we have

$$
\left(\sum_{i=1}^{n 2^{k}}\left\|n \sum_{s=1}^{n 2^{k}} a_{i s} y_{s}\right\|^{2}\right)^{1 / 2} \leq c \cdot 2^{(k+1) / 2}\left(\sum_{s=1}^{n 2^{k}}\left\|y_{s}\right\|^{2}\right)^{1 / 2}
$$

where $c$ is an absolute constant.

Now, we use an interpolation procedure that is well known in the present context (cf., e.g., [TJ, §28]). Consider the operator $R:\left(y_{s}\right)_{s=1}^{n 2^{k}} \rightarrow\left(\sum_{s=1}^{n 2^{k}} a_{i s} y_{s}\right)_{i=1}^{n 2^{k}}$. By $(11),\left\|R: \ell_{2}^{n 2^{k}}(T) \rightarrow \ell_{2}^{n 2^{k}}(T)\right\| \leq c \cdot 2^{(k+1) / 2}$, and clearly $\| R: \ell_{2}^{n 2^{k}}\left(\ell_{2}\right) \rightarrow$ $\ell_{2}^{n 2^{k}}\left(\ell_{2}\right) \|=1$. Interpolating these estimates and using the fact that, by $(\mathrm{d})$, $\ell_{2}^{n 2^{k}}\left(T_{\theta}\right)=\left(\ell_{2}^{n 2^{k}}(T), \ell_{2}^{n 2^{k}}(T)\right)_{\theta}$, we can choose $\theta_{k}$ such that

$$
\left\|R: \ell_{2}^{n 2^{k}}\left(T_{\theta_{k}}\right) \rightarrow \ell_{2}^{n 2^{k}}\left(T_{\theta_{k}}\right)\right\| \leq\left(c \cdot 2^{(k+1) / 2}\right)^{1-\theta_{k}} \leq 2 .
$$

This is equivalent to an inequality analogous to (11) and is known to imply the required distance estimate (cf., e.g., [P, Lemma 13.3] or [TJ, §27]). 
For every $m=2^{\ell}, \ell=1,2, \ldots$, set

$$
Y_{m}=\left.\left.\left.\left.T_{\theta_{1}}\right|_{J_{m / 2}} \oplus_{2} T_{\theta_{2}}\right|_{J_{m / 2^{2}}} \oplus_{2} T_{\theta_{3}}\right|_{J_{m / 2^{3}}} \oplus_{2} \cdots \oplus_{2} T_{\theta_{\ell}}\right|_{J_{m / 2^{\ell}}} \oplus_{2}\left(\sum_{k>\ell} \bigoplus T_{\theta_{k}}\right)_{2} .
$$

Observe that the codimension of $Y_{m}$ is $m-1$ and that every $m$-dimensional subspace $F$ of $Y_{m}$ satisfies $d\left(F, \ell_{2}^{m}\right) \leq 2$. A similar argument as in the proof of Lemma 13.5 in [P] shows that $\left(\sum_{k=1}^{\infty} \bigoplus T_{\theta_{k}}\right)_{2}$ is a weak Hilbert space.

Remark 2. Recall that the class of weak Hilbert spaces can be characterized by a linear behaviour of various functions associated with finite-dimensional structure of Banach spaces. Such functions are, for instance, the codimension of a nicely complemented Euclidean subspace in any $n$-dimensional subspace of $X$ (cf., e.g., [P]), or, as shown in [JP], the uniformity function of the uniform approximation property of $X$. It is well known [P] that, for examples related to the Tsirelson space, these functions have much slower growth than linear, and the same has recently been shown in [NT] for an arbitrary weak Hilbert space with an unconditional basis. Obviously, for the weak Hilbert space constructed in Theorem 2, the mentioned functions, as well as many other functions discussed in [P], which do not necessarily characterize weak Hilbert spaces, have growth as slow as for the Tsirelson space.

\section{ACKNOWLEDGMENT}

This note is a part of the author's $\mathrm{Ph}$. D. thesis prepared under the supervision of Dr. N. Tomczak-Jaegermann. The author is grateful to her for introducing him to the subject, for her extensive guidance, and for many hours of discussions.

\section{REFERENCES}

[BL] J. Bergh and J. Löfström, Interpolation spaces, Springer-Verlag, Berlin, 1976.

[B] A. Borzyszkowski, Unconditional decompositions and local unconditional structures in some subspaces of $L_{p}, 1 \leq p<2$, Studia Math. 76 (1983), 267-278.

[CS] P. Casazza and T. Shura, Tsirelson's space, Lecture Notes in Math., vol. 1363, SpringerVerlag, New York, 1963.

[JLS] W. B. Johnson, J. Lindenstrauss, and G. Schechtman, On the relation between several notations of unconditional structure, Israel J. Math. 37 (1980), 120-129.

[JP] W. B. Johnson and G. Pisier, The proportional U.A.P. characterizes weak Hilbert spaces, J. London Math. Soc. (2) 44 (1991), 525-536.

[KP] N. J. Kalton and T. Peck, Twisted sums of sequence spaces and the three space problem, Trans. Amer. Math. Soc. 255 (1979), 1-30.

[K] T. Ketonen, On unconditionality in $L_{p}$ spaces, Ann. Acad. Sci. Fenn. Ser. A I Math. Dissertationes 35 (1983), 3-42.

[NT] N. J. Nielsen and N. Tomczak-Jaegermann, Banach lattices with property $(H)$ and weak Hilbert spaces, Illinois J. Math. (to appear).

[P] G. Pisier, Volume of convex bodies and the geometry of Banach spaces, Cambridge Univ. Press, Cambridge, 1990.

[TJ] N. Tomczak-Jaegermann, Banach-Mazur distance and finite-dimensional operator ideals, Pitman Monographs Surveys Pure Appl. Math., vol. 38, Longman Sci. Tech., Harlow, and Wiley, New York.

Department of Mathematics, University of Alberta, Edmonton, Alberta, Canada T6G 2G1 